\title{
Increased resistance towards fatigability in patients with facioscapulohumeral muscular dystrophy
}

\author{
Matteo Beretta-Piccoli ${ }^{1,2}\left(\right.$ D $\cdot$ Luca Calanni $^{1} \cdot$ Massimo Negro ${ }^{1}$ (D) Giulia Ricci ${ }^{3} \cdot$ Cinzia Bettio $^{4} \cdot$ Marco Barbero $^{2}$ (1) .

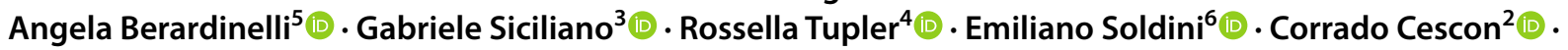 \\ Giuseppe D'Antona ${ }^{1,7}$ (D)
}

Received: 25 September 2020 / Accepted: 19 February 2021 / Published online: 1 March 2021

(c) The Author(s) 2021

\begin{abstract}
Purpose In facioscapulohumeral muscular dystrophy (FSHD) fatigue is a major complaint. We aimed to investigate whether during isometric sustained elbow flexions, performance fatigability indexes differ in patients with FSHD with respect to healthy controls.

Methods Seventeen patients with FSHD and seventeen healthy controls performed two isometric flexions of the dominant biceps brachii at $20 \%$ of their maximal voluntary contraction (MVC) for $2 \mathrm{~min}$ and then at $60 \%$ MVC until exhaustion. Muscle weakness was characterized as a percentage of predicted values. Maximal voluntary strength, endurance time and performance fatigability indices (mean frequency of the power spectrum (MNF), muscle fiber conduction velocity (CV) and fractal dimension (FD)), extracted from the surface electromyogram signal (sEMG) were compared between the two groups. Results In patients with FSHD, maximal voluntary strength was $68.7 \%$ of predicted value $(p<0.01)$. Compared to healthy controls, FSHD patients showed reduced MVC $(p<0.001 ; r=0.62)$ and lower levels of performance fatigability, characterized by reduced rate of changes in MNF $(p<0.01 ; r=0.56), \mathrm{CV}(p<0.05 ; 0.37)$ and FD $(p<0.001 ; r=0.51)$ and increased endurance time $(p<0.001 ; r=0.63)$, during the isometric contraction at $60 \%$ MVC.

Conclusion A decreased reduction in the slopes of all the considered sEMG parameters during sustained isometric elbow flexions suggests that patients with FSHD experience lower levels of performance fatigability compared to healthy controls.
\end{abstract}

Keywords Neuromuscular disease $\cdot$ Dystrophy $\cdot$ Electromyography $\cdot$ Fatigability $\cdot$ Biceps brachii

Abbreviations
FSHD Facioscapulohumeral muscular dystrophy
EMG Electromyography
MVC Maximal voluntary contraction

Communicated by Nicolas place.

Giuseppe D'Antona

gdantona@unipv.it

1 Criams-Sport Medicine Centre Voghera, University of Pavia, Voghera, Italy

2 Rehabilitation Research Laboratory 2rLab, Department of Business Economics, Health and Social Care, University of Applied Sciences and Arts of Southern Switzerland, Manno/Landquart, Switzerland

3 Department of Clinical and Experimental Medicine, University of Pisa, Pisa, Italy

4 Department of Life Sciences, University of Modena and Reggio Emilia, Modena, Italy
CIS Checklist individual strength

CV Conduction velocity

MNF Mean power frequency

FD Fractal dimension

IQR Interquartile range
5 Child Neuropsychiatry, IRCCS Mondino Foundation, Pavia, Italy

6 Research Methodology Competence Centre, Department of Business Economics, Health and Social Care, University of Applied Sciences and Arts of Southern Switzerland, Manno, Switzerland

7 Department of Public Health, Experimental and Forensic Medicine and Sport Medicine Centre Voghera, University of Pavia, via Foscolo, 13 - 27058 Voghera, Italy 


\section{Introduction}

Fatigue is known to be a common symptom in muscular dystrophies (Kalkman et al. 2005). Among the dystrophies whose genetic defects have been identified at the molecular level, facioscapulohumeral muscular dystrophy (FSHD), presents the most peculiar mutation: the FSHD gene defect is in fact not located in any protein-coding gene (Tupler and Gabellini 2004). Instead, in the majority of patients, the disease was associated with the contraction of a polymorphic region known as D4Z4 (chromosome 4q35) that is characterized by an array of tandemly repeated units of 3.3 kb (van Deutekom et al. 1993; Wijmenga et al. 1992). The primary mediator of FSHD pathology is thought to be the expression of DUX4, a gene epigenetically repressed in most somatic tissues located in each unit of the D4Z4 repeat array (Lemmers et al. 2010). In patients with FSHD, inadequate DUX4 protein expression in skeletal muscle is favored by D4Z4 chromatin relaxation (Lassche et al. 2020). Clinically, FSHD is characterized by slowly progressive weakness of the muscles of the face, shoulder, and upper arm. However, many patients also have weakness of the trunk and leg muscles; in fact, sometimes these are the most pronounced symptoms. In addition, some patients have no symptoms or only very mild symptoms (Mul et al. 2016). Progression of the disease is associated with atrophy and fatty infiltration of the muscle tissue which can be visualized on MRI (Mul et al. 2017).

In FSHD, fatigue occurs as an early leitmotif of the disease and as a disabling symptom in daily activities; in a recent qualitative study, patients described fatigue as "an overwhelming and unpredictable experience" without recognizing the underlying causes, making it difficult to cope with and thus massively affecting participation, social contacts, and quality of life (Schipper et al. 2017). Furthermore, in a survey involving 328 participants with FSHD, one of the symptoms with the highest prevalence was fatigue (93.8\%) (Hamel et al. 2019).

For the purposes of this study, fatigue will be discussed within the taxonomy proposed by Kluger et al. (2013). Specifically, fatigue is defined as a symptom or perception, characterized by feelings of tiredness and weakness, in which physical and cognitive functions are impaired because of interactions between performance fatigability and perceived fatigability. Performance fatigability refers to the decline in an objective measure of performance, such as the generation of maximal voluntary force (MVC), the ability to provide an adequate signal for voluntary muscle activation, or the involuntary twitch response to stimulation (Enoka and Duchateau 2016). To assess muscle contractility or the degree of muscle activation before and during a performance task in FSHD, several studies have been conducted using electrophysiological responses evoked by muscle or peripheral nerve stimulation. For example, Schulte-Mattler et al. (2003) described excessive fatigability during isometric ankle dorsiflexion in four patients with FSHD. Later, Schillings et al. (2007) described reduced performance fatigability after a 2-min sustained isometric MVC of the biceps brachii (BB) in 65 patients with FSHD, compared to control subjects. Recently, Bachasson et al. (2014) demonstrated similar fatigability during intermittent isometric contractions in the quadriceps femoris in 19 FSHD patients compared to controls, using femoral nerve magnetic stimulation. However, to overcome the limitations of electrical/magnetic stimulation in clinical populations (discussed in Place and Millet 2020), such as the impossibility to test neuromuscular function under physiological conditions, pain during stimulation that can lead to biased measurements, the contribution of intramuscular processes to the superimposed force during fatigue and insufficient stimulation intensity, performance fatigability can be assessed using surface electromyography (sEMG). For example, spectral parameters, muscle fiber conduction velocity (CV) or nonlinear parameters (reviewed in Rampichini et al. 2020) are suitable to be used as indirect indices of performance fatigability. Indeed, fatigability during isometric constant force contractions, can be observed by the decrease of $\mathrm{CV}$ mainly related to a decrease of intracellular $\mathrm{pH}$ (Komi and Tesch 1979). A decrease in the fractal dimension (FD) of the sEMG signal has been associated with fatigability, aging, and disease (Beretta-Piccoli et al. 2020; Goldberger et al. 2002; Arjunan and Kumar 2013). These findings suggest a potential utility of the fractal analysis of the sEMG signal as a complementary tool for assessing fatigability during a performance test.

Therefore, the primary objective of this study was to investigate whether patients fatigue differently than healthy controls (HC) during sustained isometric elbow flexions. We hypothesized that since FSHD is characterized by a transition from fast-glycolytic to slow-oxidative muscle fibres (Celegato et al. 2006), patients would show less performance fatigability compared to controls.

\section{Methods}

\section{Participants}

This cross-sectional study was performed according to the Declaration of Helsinki, with the approval of the local Ethics Committee of the University of Pisa. Seventeen patients with FSHD and seventeen HC were recruited after providing written informed consent. 
The study was part of a crowdfunding project (\#Sport4therapy) conducted at CRIAMS-Sport Medicine Centre Voghera and sponsored by the University of Pavia, aiming to identify the correct sports therapy approach in patients affected by rare neuromuscular diseases (Siciliano et al. 2019), including FSHD (Berardinelli and D'Antona 2019). Data collection began in 2013 and was completed in 2019. Inclusion criteria were age of $\geq 16$ years, a clinical or genetic diagnosis of FSHD, and enrollment in the Italian National Registry for FSHD. Exclusion criteria were wheelchair bound at selection, use of corticosteroids, severe cardiac and respiratory dysfunction, and psychological/psychiatric disorders. A diagnosis of FSHD had to be confirmed by DNA testing (Lemmers et al. 2012) at the University of Modena and Reggio Emilia (Italy). Patients with FSHD were enrolled in the study. The severity of the disease was assessed using the FSHD clinical score (Lamperti et al. 2010), which ranges from 0 , when there are no objective signs of functional impairment, to 15 , when all tested muscle groups are severely impaired and the patient is wheelchair dependent. Each section describes the functional assessment of six muscle districts that are peculiarly affected in FSHD: face (score 0-2); shoulder girdle (score 0-3); upper limbs (score 0-2); distal legs (score 0-2); pelvic girdle (score $0-5$ ); abdominal muscles (score $0-1$ ). The protocol assigns an independent score to each muscle group, providing an accurate description of the distribution of muscle weakness for each individual. The main characteristics of the patients are listed in Table 1.

\section{FSHD categories}

Patients were allocated to the four clinical categories according to the Comprehensive Clinical Evaluation Form (CCEF, Ricci et al. 2016). The CCEF classifies (1) subjects with facial and scapular girdle muscle weakness typical of FSHD (category A), (2) subjects with muscle weakness limited to the scapular girdle or facial muscles (category B), (3) asymptomatic/healthy subjects (category $\mathrm{C}$ ), and (4) subjects with a myopathic phenotype presenting clinical features not consistent with the canonical phenotype of FSHD (category D).

Since patients belonging to category A have the most peculiar signs of the disease, they were compared with the other categories, to highlight differences in the clinical variables considered.

\section{FSHD asymmetry}

Significant asymmetry of muscle involvement was previously observed in the upper extremities, showing right-sided dominance, regardless of handedness (Rijken et al. 2014). Asymmetry of muscle involvement was clinically evaluated
Table 1 Descriptive statistics of the socio-demographic and clinical variables

\begin{tabular}{|c|c|c|c|}
\hline & $n$ & Median & IQR \\
\hline \multicolumn{4}{|l|}{ Socio-demographic variables } \\
\hline \multicolumn{4}{|l|}{ Gender } \\
\hline Woman & 8 & - & - \\
\hline Man & 9 & - & - \\
\hline Age & - & 33.0 & 31.25 \\
\hline \multicolumn{4}{|l|}{ Clinical variables } \\
\hline \multicolumn{4}{|l|}{ FSHD categories } \\
\hline A & 12 & - & - \\
\hline B & 3 & - & - \\
\hline $\mathrm{C}$ & 0 & & \\
\hline $\mathrm{D}$ & 2 & - & - \\
\hline \multicolumn{4}{|l|}{ FSHD asymmetry } \\
\hline Right $>$ Left & 9 & - & - \\
\hline Right $=$ Left & 5 & - & - \\
\hline Right $<$ Left & 3 & - & - \\
\hline D4Z4 contraction $(\mathrm{kb}) /$ number of alleles & - & 27.00 & 11.50 \\
\hline$(11-19) / 1-3$ & 2 & & \\
\hline$(20-26) / 4-5$ & 7 & & \\
\hline$(27-31) / 6$ & 3 & & \\
\hline$(33-35) / 7-8$ & 3 & & \\
\hline$(36-41) / 9-10$ & 2 & & \\
\hline D4Z4 contraction $(\mathrm{kb})$ & - & 27.00 & 11.50 \\
\hline Checklist individual strength $^{\mathrm{a}}$ & - & 25.50 & 15.00 \\
\hline Severity of FSHD (clinical score) & - & 4.00 & 6.25 \\
\hline Scapular girdle involvement score & & 2.00 & 1.00 \\
\hline
\end{tabular}

${ }^{a}$ Variable with three missing values $(\mathrm{n}=14)$

FSHD facioscapulohumeral muscular dystrophy, $I Q R$ interquartile range

Categories definition see text

and a comparative analysis between patients with predominant right-sided or left-sided involvement was performed to determine if side involvement correlated with the clinical severity of the disease.

\section{Experimental procedures}

\section{Perceived fatigability}

The degree of perceived fatigability was assessed before the fatigue task using the fatigue subscale of the Checklist Individual Strength (CIS fatigue). This scale consists of eight questions regarding fatigability experienced during the previous 2 weeks; each question was scored on a 7-point Likert scale (Vercoulen et al. 1994). A total score $\geq 35$ indicates severe fatigue. The CIS fatigue has good internal consistency (Cronbach a 0.83-0.92), high discriminant validity, and 
high sensitivity to change in patients with FSHD (Kalkman et al. 2007).

\section{Performance fatigability}

The selected protocol has been shown to induce fatigue in the elbow flexors in healthy subjects and patients (BerettaPiccoli et al. 2017, 2020). Briefly, participants were asked to perform two MVCs, separated by 2 min rest, followed by a 20\% MVC contraction that lasted 2 min and a 60\% MVC that was held until the force level dropped below $90 \%$ of the target (endurance time, i.e. the time for which a subject can sustain the requested mechanical task). The two sub-maximal contractions were separated by a 5-min rest. Since arm muscles show early disability in FSHD (Derry et al. 2012; Rijken et al. 2014; Tawil 2018), the BB was selected as the affected muscle. In particular, the BB is subject to selective muscle wasting resulting in so-called 'Popeye' arms because of the contrast between atrophied perihumeral musculature and sparing of the forearm and distal deltoid muscles (Mul et al. 2016). Furthermore, because this muscle has long fibers that run parallel to the skin, high quality sEMG acquisitions, according to Barbero et al. (2012) may be recorded. Finally, sub-maximal contractions were selected because they are more representative of the intensity of activities performed during daily life.

\section{EMG and force measurements}

Myoelectric signals were recorded from the dominant BB in a monopolar configuration. Participants were seated on a height-adjustable chair with their arm positioned on an isometric ergometer (MUC1, OT-Bioelettronica, Turin, Italy), equipped with a load cell (Model TF022, CCT Transducers, Turin, Italy). The wrist was fastened to the ergometer, with the elbow flexed at $120^{\circ}$. A bi-dimensional array of 64 electrodes ( $3 \mathrm{~mm}$ diameter, $8 \times 8$ grid, $10 \mathrm{~mm}$ interelectrode spacing; model ELSCH064NM3; OT-Bioelettronica) was positioned on the BB according to Barbero et al. (2012) with the distal edge close to the cubital fossa and the midline of the array aligned with the midline of the $\mathrm{BB}$ along a line from the cubital fossa to the acromion. The ground electrode was placed on the contralateral wrist.

The torque of the elbow was measured using a torque meter operating linearly in the range $0-1000 \mathrm{Nm}$. The torque signal was amplified (MISO II; OT-Bioelettronica) and saved on a computer. The EMG signals were amplified with a variable factor ranging from 2000 to 5000 (EMGUSB2 +; OT-Bioelettronica), filtered with the hardware filter (10-500 $\mathrm{Hz}$ bandpass) and then with an offline Butterworth anti-causal bandpass filter (2nd order-20-400 Hz bandpass) and sampled together with the torque signal at $2048 \mathrm{~Hz}$ using a 16-bit A/D converter, with $5 \mathrm{~V}$ dynamic range, and stored on a computer. The torque signal was displayed on a screen, as real-time biofeedback.

\section{Assessment of muscle weakness}

Normative data on the maximal voluntary isometric force exerted by healthy subjects at $90^{\circ}$ elbow flexion were retrieved from the study by Meldrum et al. (2007). The value was then corrected for a $60^{\circ}$ elbow flexion using the following predictive equation from Bober et al. (2002):

$Y(\%)=55.49+0.88 x+0.004 x^{2}-0.0001 x^{3}$,

where $Y$ is the estimated peak torque, and $x$ is the specific joint angle.

\section{Signal processing}

The channels used for CV estimation were selected based on visual inspection of individual differential signals, along one of the array columns, as described previously (BerettaPiccoli et al. 2017; Fig. 1), and their number typically ranged from four and seven (Farina et al. 2004). CV was estimated using a multichannel algorithm (Farina and Merletti 2003) on single differential signals based on matching temporally and spatially filtered signals, using non-overlapping signal epochs of $1 \mathrm{~s}$, on the selected channels. Changes in CV during fatiguing contractions have a profound impact on the shape of the motor unit (MU) action potential waveform and therefore on the amplitude and spectral variables extracted from the sEMG signal. Estimation of the $\mathrm{CV}$ slope (i.e. rate of change), might be useful to characterize the peripheral components of muscle fatigue during an isometric task (Merletti and Farina 2016) and this variable may be considered as one of the most robust EMG fatigue indices (Kollmitzer et al. 1999; Linssen et al. 1993; Rainoldi et al. 2001; Dedering et al. 2000).

Each of the selected signal epochs was used to estimate the mean frequency of the power spectrum (MNF) and the fractal dimension of the sEMG signal (FD); these variables were averaged over all selected channels. MNF (a parameter used to quantify the changes in the spectral content of the sEMG signal based on the Fourier transform) was computed offline with a numerical algorithm (Merletti et al. 1990) using the following calculation formula (Rampichini et al. 2020):

$M N F=\frac{\int_{f 1}^{f 2} f \cdot P S(f) \cdot d f}{\int_{f 1}^{f 2} P S(f) \cdot d f}$,

where PS(f) is the sEMG power spectrum calculated by Fourier transform, and $f 1$ and $f 2$ determine the bandwidth of the surface electromyography $(f 1=$ lowest frequency and 


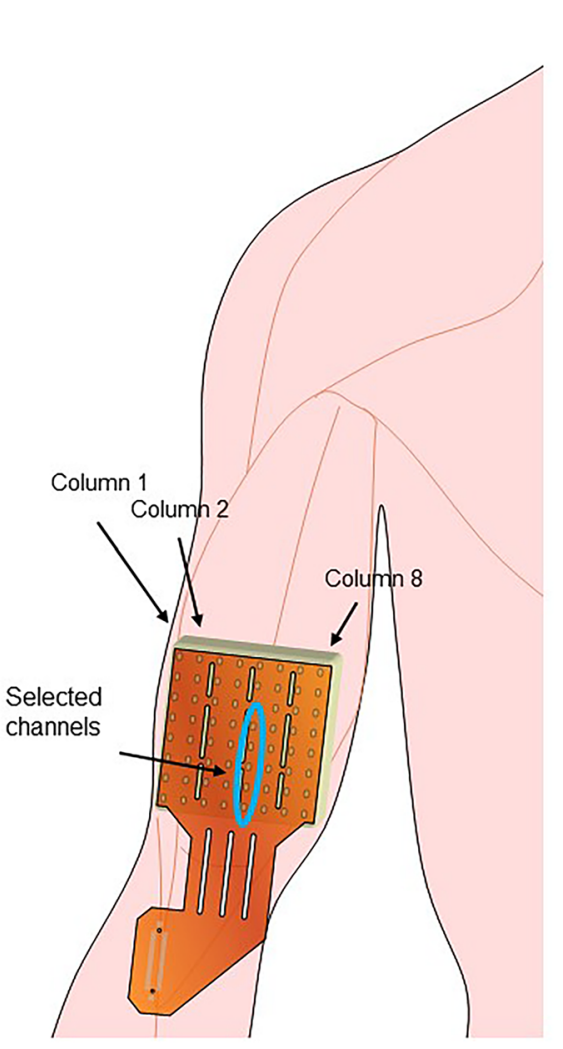

Fig. 1 Representation of the position of sEMG array on the biceps brachii muscle. An example of EMG signals detected in single differential mode from each column of a FSHD patient during an isometric elbow flexion at $60 \% \mathrm{MVC}$ is shown on the right panel. The innerva-

$f 2=$ highest frequency of the bandwidth). MNF is related to changes in muscle fiber $\mathrm{CV}$ and subsequent changes in intracellular action potential duration (Bigland-Ritchie et al. 1981). It has been shown during static contractions that MNF shifts to lower frequencies with increasing fatigue (Lindström et al. 1977; Merletti et al. 1990; Merletti and Lo Conte 1997; Viitasalo and Komi 1977), due to decreased $\mathrm{CV}$ as a result of local metabolic changes in the working muscle, mainly $\mathrm{H}^{+}$and $\mathrm{K}^{+}$distribution across the sarcolemma (Dimitrova and Dimitrov 2003; Masuda et al. 1983). However, modifications of MU action potential shape, MU firing rate and synchronization may also contribute to MNF changes (Bigland-Ritchie and Woods 1984; Brody et al. 1991; Gabriel and Kamen 2009).

FD was estimated using the box-counting method as previously reported (Gitter and Czerniecki 1995). Briefly, a grid of square boxes is used to cover the EMG signal, and the number of boxes through which the signal passes is counted. If one decreases the side of the boxes in a dichotomous process, the number of boxes counted increases exponentially. However, if one plots the logarithm of the number of boxes required to cover the signal tion zone can be identified by the $\mathrm{V}$ shape of the signals. The selected channels for muscle fiber estimation are located in the distal portion of column 5, where the pure propagation of motor unit action potentials is visible between the innervation zone and the distal tendon

against the logarithm of the inverse of the box area, one obtains an approximately linear relationship. The slope of the interpolation line (estimated by the least mean squared method) is the FD (Mesin et al. 2009). Therefore, the following expression defines the FD of the sEMG signal:

$\mathrm{FD}=\log N / \log (1 / L)$,

where $N$ is the number of boxes required to cover the signal, $L$ is the box side, and the ratio is the slope of the interpolation line.

FD has been proposed as an index to monitor changes in the sEMG signal during an isometric fatigue task (BerettaPiccoli et al. 2015; Boccia et al. 2016; Mesin et al. 2009). Although the use of non-linear analysis of the sEMG signal is desirable, as it is more sensitive than spectral analysis for assessing performance fatigability(Farina et al. 2002), it is difficult to relate these parameters to physiological changes in muscle properties resulting from fatigue. Nevertheless, Mesin et al. (2016) demonstrated an inverse relationship between FD and MU synchronization and a positive relationship with the MU firing rate during simulated isometric fatigue contractions. 
Performance fatigability was indirectly quantified as the slopes of the considered sEMG variables during endurance contraction.

\section{Statistical analysis}

Descriptive statistics were used to represent the variables included in the comparative analysis; categorical variables were described by frequency distributions, while continuous variables were described by synthetic indicators (median and interquartile range, IQR). At the bivariate level, the analyses were conducted using nonparametric statistical indicators and tests to account for the small sample size and generalized non-normality of the distributions. Linear regression over time was applied to MNF, CV and FD to extract initial values and slopes, the latter normalized with respect to their initial values.

Differences in the sEMG measures between FSHD patients and $\mathrm{HC}$, as well as differences in the continuous clinical variables related to FSHD categories and asymmetry, were assessed using the Mann-Whitney U test. The Cohen's r estimate of effect size was calculated using (Cohen 1988):

$r=\frac{|z|}{\sqrt{N}}$,

where $\mathrm{z}$ is the value obtained in the Mann-Whitney $\mathrm{U}$ test and $\mathrm{N}$ the total number of observations, as suggested by Fritz et al. (2011). Cohen's guidelines outline that the effect size is low if the value of $r$ varies around 0.1 , medium if $r$ varies around 0.3 , and large if $r$ varies more than 0.5 (Coolican 2009). Eventually, the Wilcoxon signed-rank test was run to determine differences between predicted and measured maximal voluntary strength in patients with FSHD, and whether the slopes of the EMG parameters at 20\% MVC differed from zero. The statistical significance was set at $\alpha=0.05$. All statistical analyses have been carried out with Stata/IC 16.0 (StataCorp, College Station, Texas, USA).

\section{Results}

\section{Socio-demographic and clinical variables}

Twelve out of 17 patients belonged to category "A", according to Ricci et al. (2016), presenting facial and scapular girdle muscle weaknesses. More accentuated muscle weakness on the right side was observed in nine patients, eight of whom were right-handed (Table 1, "Right > Left" category), while it was more accentuated on the left side for three patients and was equally distributed in five of them. The number of patients per $\mathrm{D} 4 \mathrm{Z} 4$ contraction/number of repeats is described in Table 1. The median clinical score assessing the severity of FSHD was 4 [IQR = 6.25], while the median scapular girdle involvement score was 2 [IQR $=1]$. The difference in median age between patients with FSHD and HC was not statistically significant.

\section{Perceived fatigability}

Perceived fatigability, assessed in patients with FSHD using the CIS fatigue subscale, was reported as mild (25.5, $[\mathrm{IQR}=15])$.

\section{Performance fatigability}

The time courses of MNF, CV and FD during 20\% and 60\% MVC are shown in Fig. 2 for a representative subject. The hypothesis test highlighted some statistically significant differences between the two groups, which are shown in Fig. 3: (1) the initial values of MNF and FD at $20 \%$ MVC yielded higher values in the FSHD group ( $p 0.05$ and $\mathrm{p} 0.001$, respectively), while their negative slopes showed a less steep decline at $60 \%$ MVC ( $p$ 0.01). (2) A similar decrease was also observed for the negative slope of $\mathrm{CV}$ at $60 \% \mathrm{MVC}$ in the FSHD group ( $p 0.05$ ). At $20 \%$ MVC, the slope of MNF was not different from 0 in the FSHD group $(p=0.14)$, and in both groups for $\mathrm{CV}$ ( $p=0.08$ and $p=0.53$, respectively). In addition, exerted force was lower in the FSHD group, while endurance time was longer compared to the HC group $(p=0.001)$. Effect size analysis revealed high values for MNF slope and FD slope during 60\% MVC contraction ( $\mathrm{r}$ estimates and 0.56 and 0.51 , respectively), FD initial value during low-level contraction $(r=0.61)$, and maximum voluntary force $(r=0.62)$ and endurance time $(r=0.63)$. Moreover, the initial values of MNF at $20 \%$ MVC and the slope of $\mathrm{CV}$ at $60 \%$ MVC showed a medium effect (r-estimates and 0.33 and 0.37 , respectively). A smaller effect size was found for the remaining parameters.

\section{Muscle weakness in FSHD patients}

The median MVC at $60^{\circ}$ of isometric elbow flexion was $19.79[\mathrm{IQR}=10.87] \mathrm{kg}$, while the predicted MVC in heathy subjects was 28.82 [IQR $=11.76] \mathrm{kg}$. Consequently, the maximal strength was $31.3 \%$ significantly lower than the predicted value $(p<0.01)$. 

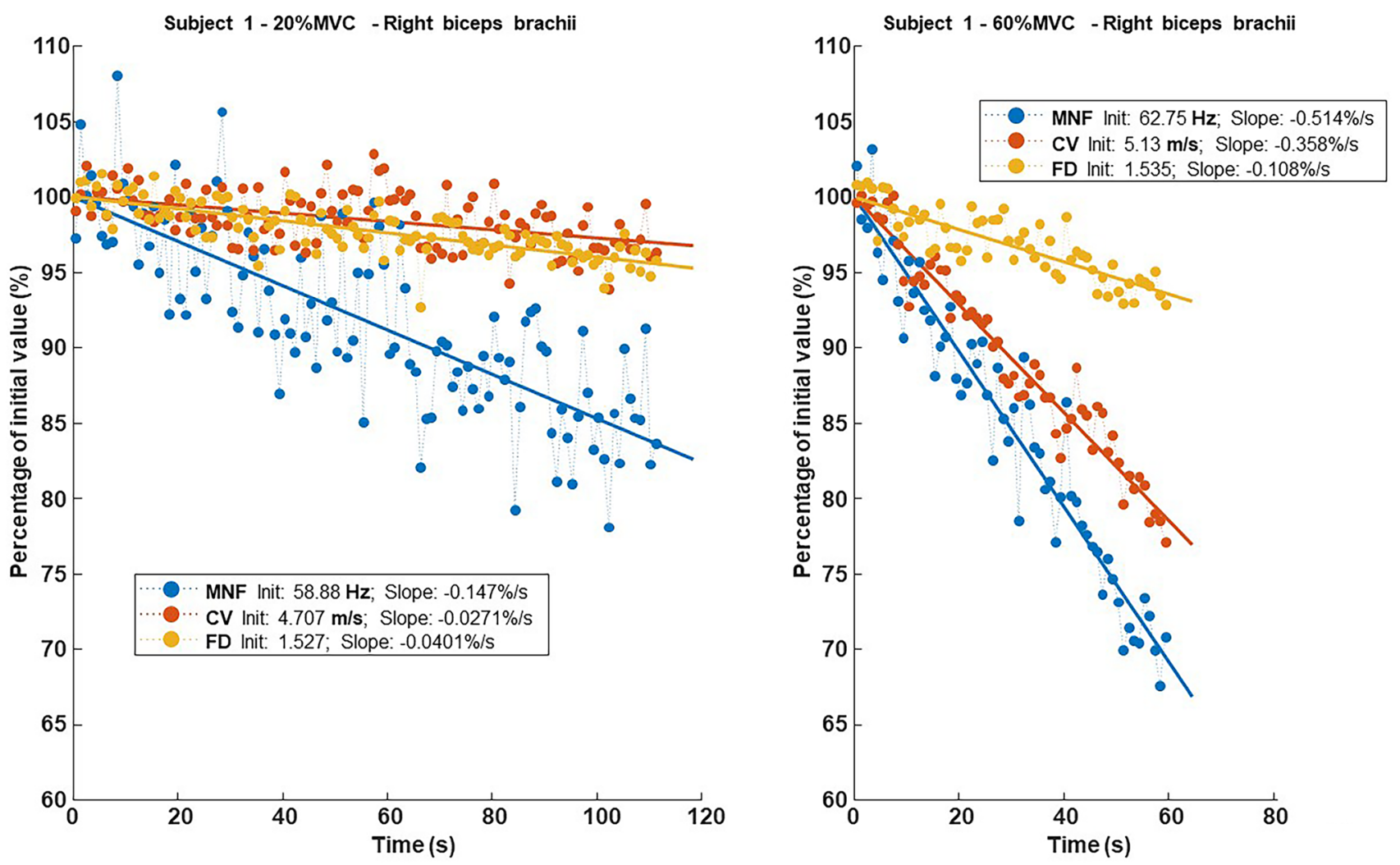

Fig. 2 Time course of mean power spectrum frequency (MNF), muscle fiber conduction velocity (CV) and fractal dimension (FD) for a representative patient with FSHD. Surface EMG signals were

acquired from the biceps brachii using bi-dimensional arrays during isometric contractions at $20 \%$ and $60 \%$ MVC. Data are normalized with respect to their initial values

\section{Differences in clinical variables related to FSHD category and asymmetry}

Because of the small sample size, for a robust assessment of differences in clinical variables, FSHD category and asymmetry were recoded into binary variables: "A" and "Not A", and "Right > Left" and "Not Right > Left" for FSHD categories and asymmetry, respectively. D4Z4 contraction length showed no statistically significant differences between FSHD categories, whereas patients with more severe right-sided muscle (category "Right $>$ Left") reported a significantly lower FSHD severity $(z=-2.156$; $p<0.05)$.

\section{Discussion}

In this study, sEMG parameters, known as indirect indices of performance fatigability, were examined in FSHD subjects and $\mathrm{HC}$ during isometric fatigue contractions of the elbow flexors. The results showed significant differences in all parameters considered between the two groups; in particular, FSHD patients presented lower MVC and lower levels of performance fatigability (i.e. reduced slope of MNF, CV and FD), and increased time to exhaustion during isometric fatigue contraction at $60 \%$ MVC.

\section{Muscle weakness, strength, and endurance time}

In agreement with previous studies, the results presented a fairly consistent picture, characterized by the inability of patients with FSHD to exert maximal strength comparable to the one of HC (Bachasson et al. 2014; Doix et al. 2017; Kalkman et al. 2007; Turki et al. 2012). This finding is corroborated by an increased predicted muscle weakness experienced by patients with FSHD. A recent study by Lassche et al. (2020) hypothesized that muscle weakness in FSHD is not caused by alterations in sarcomeric contractility, or excitation-contraction coupling (Vandebrouck et al. 2002), but is due to DUX4-induced toxicity and consequential loss of muscle fibers or to an unknown impaired metabolic cause.

In addition, the patients' endurance time was significantly longer than in HC, suggesting an increased resistance to the development of fatigue (Fig. 3a), probably for several reasons: 


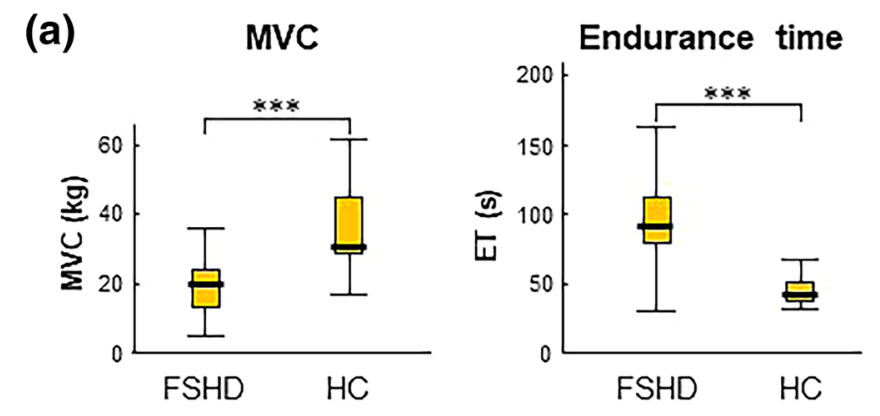

(b)

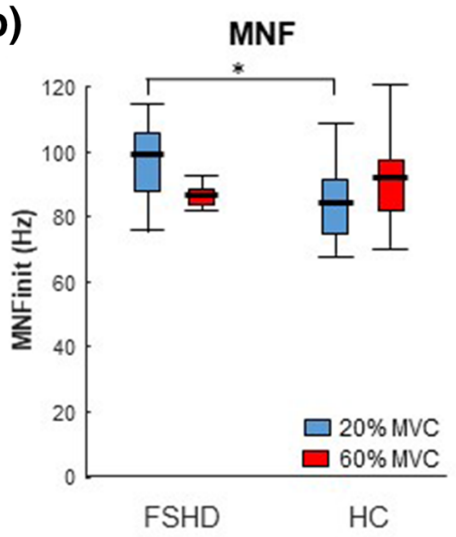

(c)

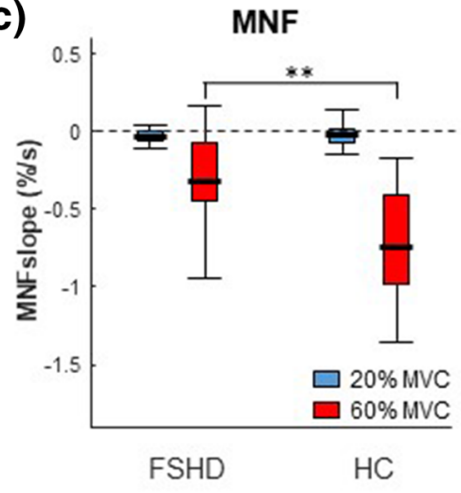

CV

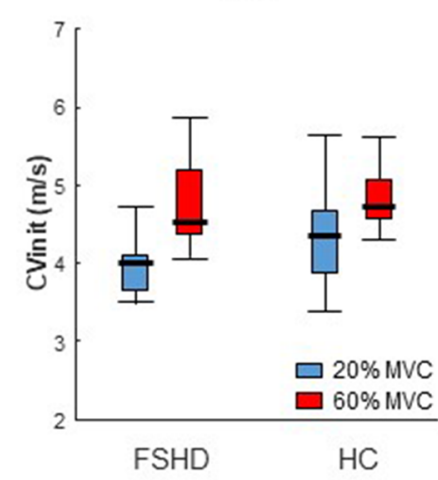

CV

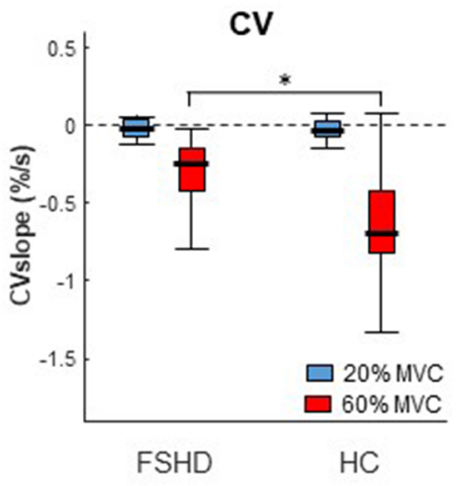

${ }^{*} p<0.05,{ }^{* *} p<0.01,{ }^{* \star *} p<0.001$
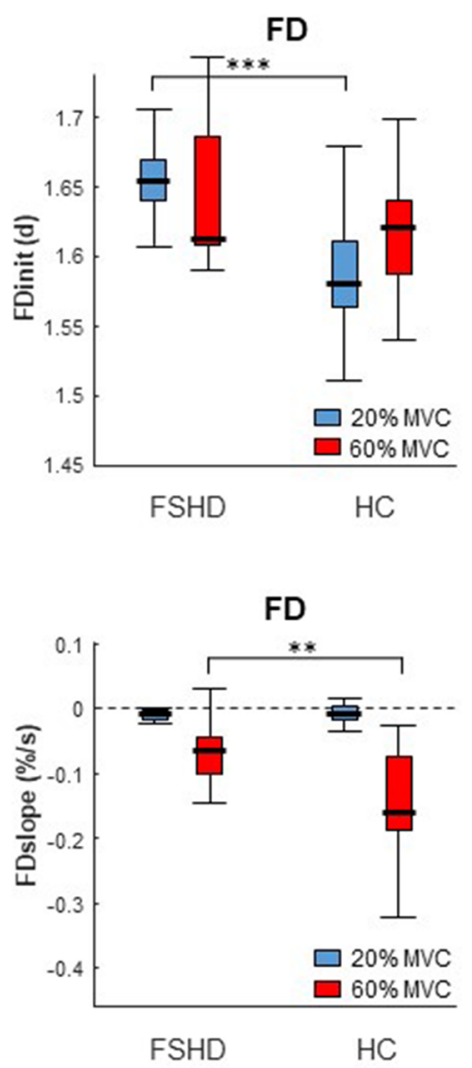

$\mathrm{MNF}$, mean frequency; $\mathrm{CV}$, conduction velocity; $\mathrm{FD}$, fractal dimension. The slopes were normalized with respect to their initial values.

Fig. 3 Box-and-whisker plots of a maximal voluntary contraction (MVC) of isometric elbow flexions, and endurance time at $60 \%$ MVC; $\mathbf{b}$ initial values and $\mathbf{c}$ slopes of mean power spectrum frequency (MNF), conduction velocity (CV) and fractal dimension (FD)

First, patients with FSHD may exhibit fibrosis and fatty infiltration (Friedman et al. 2012) which are associated with changes in muscle composition and, particularly from fastglycolytic (type IIX) to slow-oxidative (type I) muscle fibers (Celegato et al. 2006). Indeed, these structural alterations evaluated by MRI, have been associated with a significantly decreased phosphocreatine/ATP ratio (Janssen et al. 2014), during the $20 \%$ and $60 \%$ MVC. Slopes were normalized with respect to their initial values. HC, healthy controls. Asterisks denote statistical significance at $* \mathrm{p}<0.05, * * \mathrm{p}<0.01, * * * \mathrm{p}<0.005$

histological findings of biopsies showing more dominant type I fibers (Dubowitz et al. 2020), and loss of muscle strength. Second, weaker participants have been less fatigable than stronger ones (Hunter and Enoka 2001) because intramuscular pressure is lower, the blood occlusion is also lower (Zwarts and Arendt-Nielsen 1988) and negative feedback from afferent groups III and IV is reduced (Gandevia 
2001). Reduced fatigability in patients with FSHD with respect to HC was also described in Doix et al. (2017), before and after short-term neuromuscular electrical stimulation training of the anterior tibialis muscle, which may be affected similarly to the BB in earlier stages of the disease (Olsen et al. 2006; Dorobek et al. 2013).

\section{Performance fatigability}

Interestingly, the fatigability indices extracted from the sEMG signal suggested an increased resistance to fatigue in the FSHD patients (Fig. 3b). Indeed, the reduction in the normalized slope of MNF, CV and FD during high-level fatigue contraction, a sign of an increase in performance fatigability (Merletti and Farina 2016; Beretta-Piccoli et al. 2017 and 2020), was significantly lower in patients with FSHD compared to HC. Similar results were reported by Schillings et al. (2007), who described lower peripheral fatigue in FSHD patients compared to controls, which was directly related to lower maximal effort and blood flow occlusion compared to controls. Moreover, the lower decrease in CV slope may also be explained by a larger contribution of fatigue-resistant type I muscle fibers, a well-known feature of FSHD (D'Antona et al. 2007).

However, the reduction in fatigability also affects the slope of MNF (which is related to both a reduction in muscle fibers CV and an increase in MU firing rate and synchronization Bigland-Ritchie and Woods 1984; Brody et al. 1991; Gabriel and Kamen 2009), and the slope of FD (which is related to an increase in MU firing rate and synchronization (Rampichini et al. 2020). It is, therefore, reasonable to hypothesize that the reduced fatigability in FSHD is also due to central factors, as also suggested by Schillings et al. (2007).

On the contrary, during the $20 \%$ MVC contraction, the normalized slopes of the fatigability indices did not differ, suggesting that the level of contraction was not fatiguing (confirmed by the slopes of $\mathrm{CV}$, which did not differ from 0 in the two groups) and hypothesizing that there is a greater recruitment of type I muscle fibers than type II in FSHD patients (Fig. 3b).

\section{Initial values}

During low-level contraction, which was non fatiguing for both groups, increased initial values of MNF and FD were observed in patients with FSHD (Fig. 3c). This result is not surprising, since a greater impact of the physiological factors affect the initial values of MNF. Unexpectedly, no significant difference was observed in muscle fiber $\mathrm{CV}$, although reduced CV levels have been reported in previous studies in other neuromuscular disorders (Naumann and Reiners 1996; Al-Ani et al. 2001; Butugan et al. 2014).
Probably, this is related to a heterogeneous control group, in which individuals have different fatigue patterns. On the contrary, the result of the initial values of FD of the sEMG signal, which quantitatively indicates the chaotic behavior of the signal, and is related to the degree of interference (Mesin et al. 2009), suggests an increased complexity of the signal compared to the controls, especially at $20 \%$ MVC. Several studies determined that FD can be used to quantify the complexity of MU recruitment patterns (Arjunan and Kumar 2016). Since it is well known that MU recruitment is impaired in neuromuscular disorders and more MUs are recruited in myopathies even at low forces (Sanders et al. 1996), an increase in signal complexity may be hypothesized. Furthermore, Derry et al. (2012) observed a more complex MU action potential morphology during low-level (10-20\% MVC) isometric contractions of the BB in FSHD patients compared to controls. Eventually, they showed that as the disease progressed and muscle fibers were increasingly lost due to the degenerative process, complexity increased.

\section{Influence of FSHD category and asymmetry over fatigability}

Surprisingly, no statistically significant differences in clinical variables were detected between FSHD categories classified as A or not-A, suggesting that the two groups are comparable from a clinical point of view. In particular, the fact that the D4Z4 array does not differ between the categories seems to underline a labile relationship with the clinical picture and even that the size of the deletion is not sufficient to tell whether a patient with eight repeated units belongs to A or not-A category. The two categories considered share common pathophysiological traits, at least as far as fatigue is concerned. For instance, the most conclusive common event is loss of strength and a fast-to-slow shift of muscle fiber type composition.

The results regarding asymmetry, which is a very common feature in FSHD, unexpectedly showed that patients with more accentuated muscle weakness on the right side have a better clinical picture, highlighted by a lower median severity score compared to those with more left sided or equal right/left involvement.

The prevalence of right-sided involvement is consistent with previous findings and has been associated with mechanical factors and, in particular, with preferential use of the right side by right-handed individuals (Brouwer et al. 1992; Tasca et al. 2014).

\section{Limitations}

A limitation of this study is related to the model used to fit time-dependent changes in EMG parameters during the 
high-level isometric contractions. Linear trend analysis was used. However, changes in MNF and CV may also follow an exponential trend during sub-maximal contractions (Merletti et al., 1991). Here, a linear model greatly simplified the analysis, and inspection of the data showed that the assumption of linear trends was reasonable for all parameters. Moreover, as fatigability assessment is task dependent (Enoka 1995), protocol specifications are known to influence findings and underlying mechanisms of fatigue. Furthermore, the majority of studies on fatigability in FSHD patients have been conducted using electrical stimulation, thus the results may not be comparable. In addition, we evaluated fatigability in the dominant BB only, which may not represent the disease condition of the whole individual. Although the BB is often one of the first muscles to manifest signs of weakness in FSHD, it is not excluded that muscles of the shoulder girdle with a more complex architecture (e.g. the trapezius) may be a better choice for evaluating early structural changes. Then, we cannot exclude phenomena of compensation of muscle groups that may affect the developed force. Moreover, our results are based on a small group of patients, thus the statistical power is rather low, especially for the comparisons between patients. Finally, the rate of perceived exertion after the tasks was not measured, so it was not possible to perform a correlation analysis between the state level of perceived and performance fatigability.

\section{Conclusions}

In summary, we reported impaired neuromuscular function in FSHD compared to HC, due to muscle weakness, which caused patients to exert lower MVC and reduced fatigability, as evidenced by longer endurance time and a lower decrease in the slopes of all sEMG fatigability indices considered during sustained isometric elbow flexions.

Further studies need to be conducted to evaluate performance fatigability in the FSHD subcategories and also to investigate the patients' fatigue induced by functional exercise unrelated to individual MVC (e.g. walking, sitto-stand transfer) to clarify the impact of fatigue on their activities of daily living.

Acknowledgements The authors wish to thank all the patients and their families for their support and Dr. Maria Douvli Smith for English language editing. This study was supported by the Crowdfunding \#sport4therapy and the French Muscular Dystrophy Association (AFMTéléthon) grant 17191 to GDA.

Author contributions GDA, GS, RT, and AB conceived and designed research. LC, MN, and GR performed the data collection. RT and CB conducted the gene experiments. MBP, LC, MN, CC, ES, and GDA analyzed data. MBP, GS, B, RT, and GDA wrote the manuscript. All authors read and approved the manuscript.
Funding Open access funding provided by Università degli Studi di Pavia within the CRUI-CARE Agreement.. This study was supported by the Crowdfunding \#sport4therapy and AFM grant 17191 to GDA.

Availability of data and materials Under motivated request to GDA.

Code availability Not applicable.

\section{Declarations}

Conflict of interest None of the authors has any conflict of interest to disclose.

Ethics approval The study was approved by the local Ethics Committee of the University of Pisa.

Consent to participate All subjects gave their written informed consent to participate in this study, which was conducted according to the Declaration of Helsinki.

Open Access This article is licensed under a Creative Commons Attribution 4.0 International License, which permits use, sharing, adaptation, distribution and reproduction in any medium or format, as long as you give appropriate credit to the original author(s) and the source, provide a link to the Creative Commons licence, and indicate if changes were made. The images or other third party material in this article are included in the article's Creative Commons licence, unless indicated otherwise in a credit line to the material. If material is not included in the article's Creative Commons licence and your intended use is not permitted by statutory regulation or exceeds the permitted use, you will need to obtain permission directly from the copyright holder. To view a copy of this licence, visit http://creativecommons.org/licenses/by/4.0/.

\section{References}

Al-Ani FS, Hamdan FB, Shaikhly KI (2001) In situ measurements of muscle fiber conduction velocity in Duchenne muscular dystrophy. Saudi Med J 22(3):259-261

Arjunan SP, Kumar DK (2013) Age-associated changes in muscle activity during isometric contraction. Muscle Nerve 47(4):545549. https://doi.org/10.1002/mus.23619

Arjunan SP, Kumar DK (2016) Fractals and Electromyograms. In: The Fractal Geometry of the Brain. Springer, pp 445-455

Bachasson D, Temesi J, Bankole C, Lagrange E, Boutte C, Millet GY, Verges S, Levy P, Feasson L, Wuyam B (2014) Assessement of quadriceps strength, endurance and fatigue in FSHD and CMT: benefits and limits of femoral nerve magnetic stimulation. Clin Neurophysiol 125(2):396-405. https://doi.org/10.1016/j.clinp h.2013.08.001

Barbero M, Rainoldi A, Merletti R (2012) Atlas of muscle innervation zones. Springer, New York

Berardinelli A, D’Antona G (2019) Physiological aspects of muscular adaptations to training translated to neuromuscular diseases. Acta Myol 38(4):197-206

Beretta-Piccoli M, Cescon C, Barbero M, Villiger M, Clijsen R, Kool J, Kesselring J, Bansi J (2020) Upper and lower limb performance fatigability in people with multiple sclerosis investigated through surface electromyography: a pilot study. Physiol Meas 41(2):025002. https://doi.org/10.1088/1361-6579/ab6f54 
Beretta-Piccoli M, D’Antona G, Barbero M, Fisher B, Dieli-Conwright CM, Clijsen R, Cescon C (2015) Evaluation of central and peripheral fatigue in the quadriceps using fractal dimension and conduction velocity in young females. PLoS ONE 10(4):e0123921. https ://doi.org/10.1371/journal.pone.0123921

Beretta-Piccoli M, D’Antona G, Zampella C, Barbero M, Clijsen R, Cescon C (2017) Test-retest reliability of muscle fiber conduction velocity and fractal dimension of surface EMG during isometric contractions. Physiol Meas 38(4):616-630. https://doi. org/10.1088/1361-6579/aa614c

Bigland-Ritchie B, Donovan EF, Roussos CS (1981) Conduction velocity and EMG power spectrum changes in fatigue of sustained maximal efforts. J Appl Physiol 51(5):1300-1305. https://doi. org/10.1152/jappl.1981.51.5.1300

Bigland-Ritchie B, Woods JJ (1984) Changes in muscle contractile properties and neural control during human muscular fatigue. Muscle Nerve 7(9):691-699. https://doi.org/10.1002/mus.88007 0902

Bober T, Kulig K, Burnfield JM, Pietraszewski B (2002) Predictive torque equations for joints of the extremities. Acta Bioeng Biomech 4(2):49-60

Boccia G, Dardanello D, Beretta-Piccoli M, Cescon C, Coratella G, Rinaldo N, Barbero M, Lanza M, Schena F, Rainoldi A (2016) Muscle fiber conduction velocity and fractal dimension of EMG during fatiguing contraction of young and elderly active men. Physiol Meas 37(1):162-174. https://doi. org/10.1088/0967-3334/37/1/162

Brody LR, Pollock MT, Roy SH, De Luca CJ, Celli B (1991) pHinduced effects on median frequency and conduction velocity of the myoelectric signal. J Appl Physiol 71(5):1878-1885. https:// doi.org/10.1152/jappl.1991.71.5.1878

Brouwer OF, Padberg GW, van der Ploeg RJ, Ruys CJ, Brand R (1992) The influence of handedness on the distribution of muscular weakness of the arm in facioscapulohumeral muscular dystrophy. Brain 115(Pt 5):1587-1598. https://doi.org/10.1093/brain/115.5.1587

Butugan MK, Sartor CD, Watari R, Martins MCS, Ortega NR, Vigneron VA, Sacco IC (2014) Multichannel EMG-based estimation of fiber conduction velocity during isometric contraction of patients with different stages of diabetic neuropathy. J Electromyogr Kinesiol 24(4):465-472

Celegato B, Capitanio D, Pescatori M, Romualdi C, Pacchioni B, Cagnin S, Vigano A, Colantoni L, Begum S, Ricci E, Wait R, Lanfranchi G, Gelfi C (2006) Parallel protein and transcript profiles of FSHD patient muscles correlate to the D4Z4 arrangement and reveal a common impairment of slow to fast fibre differentiation and a general deregulation of MyoD-dependent genes. Proteomics 6(19):5303-5321. https://doi.org/10.1002/pmic.200600056

Cohen, J (1988) Statistical power analysis for the behavioral sciences, 2nd Edition. Routledge. doi: https://doi.org/10.4324/9780203771 587

Coolican H (2009) Research methods and statistics in psychology. Hodder, London. https://doi.org/10.4324/9780203769669

D'Antona G, Brocca L, Pansarasa O, Rinaldi C, Tupler R, Bottinelli R (2007) Structural and functional alterations of muscle fibres in the novel mouse model of facioscapulohumeral muscular dystrophy. J Physiol 584(Pt 3):997-1009. https://doi.org/10.1113/jphys iol.2007.141481

Dedering A, Roos af Hjelmsäter M, Elfving B, Harms-Ringdahl K, Németh G (2000) Between-days reliability of subjective and objective assessments of back extensor muscle fatigue in subjects without lower-back pain. J Electromyogr Kinesiol 10(3):151-158. https://doi.org/10.1016/s1050-6411(00)00009-2

Derry KL, Venance SL, Doherty TJ (2012) Decomposition-based quantitative electromyography in the evaluation of muscular dystrophy severity. Muscle Nerve 45(4):507-513. https://doi.org/10.1002/ mus. 22341
Dimitrova NA, Dimitrov GV (2003) Interpretation of EMG changes with fatigue: facts, pitfalls, and fallacies. J Electromyogr Kinesiol 13(1):13-36. https://doi.org/10.1016/s1050-6411(02)00083-4

Doix AM, Roeleveld K, Garcia J, Lahaut P, Tanant V, FournierMehouas M, Desnuelle C, Colson SS, Sacconi S (2017) ShortTERM neuromuscular electrical stimulation training of the tibialis anterior did not improve strength and motor function in facioscapulohumeral muscular dystrophy patients. Am J Phys Med Rehabil 96(4):e56-e63. https://doi.org/10.1097/PHM.0000000000000705

Dorobek M, Szmidt-Sałkowska E, Rowińska-Marcińska K, Gaweł M, Hausmanowa-Petrusewicz I (2013) Relationships between clinical data and quantitative EMG findings in facioscapulohumeral muscular dystrophy. Neurol Neurochir Pol 47(1):8-17

Dubowitz V, Sewry CA, Oldfors A (2020) Muscular dystrophies and allied disorders V: facioscapulohumeral dystrophy, myotonic dystrophy, oculopharyngeal muscular dystrophy. In Muscle biopsy e-book: a practical approach. Elsevier Health Sciences, pp 300-311

Enoka RM (1995) Mechanisms of muscle fatigue: central factors and task dependency. J Electromyogr Kinesiol 5(3):141-149. https:// doi.org/10.1016/1050-6411(95)00010-w

Enoka RM, Duchateau J (2016) Translating fatigue to human performance. Med Sci Sport Exer 48(11):2228-2238. https://doi. org/10.1249/mss.0000000000000929

Farina D, Cescon C, Merletti R (2002) Influence of anatomical, physical, and detection-system parameters on surface EMG. Biol Cybern 86(6):445-456. https://doi.org/10.1007/s0042 2-002-0309-2

Farina D, Merletti R (2003) A novel approach for estimating muscle fiber conduction velocity by spatial and temporal filtering of surface EMG signals. IEEE Trans Biomed Eng 50(12):1340-1351. https://doi.org/10.1109/TBME.2003.819847

Farina D, Merletti R, Enoka RM (2004) The extraction of neural strategies from the surface EMG. J Appl Physiol 96(4):1486-1495. https://doi.org/10.1152/japplphysiol.01070.2003

Friedman SD, Poliachik SL, Carter GT, Budech CB, Bird TD, Shaw DW (2012) The magnetic resonance imaging spectrum of facioscapulohumeral muscular dystrophy. Muscle Nerve 45(4):500 506. https://doi.org/10.1002/mus.22342

Fritz CO, Morris PE, Richler JJ (2011) Effect size estimates: current use, calculations, and interpretation. J Exp Psychol Gen 141(1):2. https://doi.org/10.1037/a0024338

Gabriel DA, Kamen G (2009) Experimental and modeling investigation of spectral compression of biceps brachii SEMG activity with increasing force levels. J Electromyogr Kinesiol 19(3):437-448. https://doi.org/10.1016/j.jelekin.2007.10.009

Gandevia SC (2001) Spinal and supraspinal factors in human muscle fatigue. Physiol Rev

Gitter JA, Czerniecki MJ (1995) Fractal analysis of the electromyographic interference pattern. J Neurosci Met 58(1-2):103-108

Goldberger AL, Amaral LA, Hausdorff JM, Ivanov P, Peng CK, Stanley HE (2002) Fractal dynamics in physiology: alterations with disease and aging. PNAS 99(Suppl 1):2466-2472. https://doi. org/10.1073/pnas.012579499

Hamel J, Johnson N, Tawil R, Martens WB, Dilek N, McDermott MP, Heatwole C (2019) Patient-reported symptoms in facioscapulohumeral muscular dystrophy (PRISM-FSHD). Neurology 93(12):e1180-e1192. https://doi.org/10.1212/WNL.0000000000 008123

Hunter SK, Enoka RM (2001) Sex differences in the fatigability of arm muscles depends on absolute force during isometric contractions. J Appl Physiol 91(6):2686-2694. https://doi.org/10.1152/ jappl.2001.91.6.2686

Janssen BH, Voet NB, Nabuurs CI, Kan HE, de Rooy JW, Geurts AC, Padberg GW, van Engelen BG, Heerschap A (2014) Distinct 
disease phases in muscles of facioscapulohumeral dystrophy patients identified by MR detected fat infiltration. PLoS ONE 9(1):e85416

Kalkman JS, Schillings ML, van der Werf SP, Padberg GW, Zwarts MJ, van Engelen BG, Bleijenberg G (2005) Experienced fatigue in facioscapulohumeral dystrophy, myotonic dystrophy, and HMSNI. J Neurol Neurosurg Psychiatry 76(10):1406-1409. https://doi. org/10.1136/jnnp.2004.050005

Kalkman JS, Schillings ML, Zwarts MJ, van Engelen BG, Bleijenberg $\mathrm{G}$ (2007) The development of a model of fatigue in neuromuscular disorders: a longitudinal study. J Psychosom Res 62(5):571-579. https://doi.org/10.1016/j.jpsychores.2006.11.014

Kluger BM, Krupp LB, Enoka RM (2013) Fatigue and fatigability in neurologic illnesses: proposal for a unified taxonomy. Neurology 80(4):409-416. https://doi.org/10.1212/WNL.0b013e31827f07be

Kollmitzer J, Ebenbichler GR, Kopf A (1999) Reliability of surface electromyographic measurements. Clin Neurophysiol 110(4):725734. https://doi.org/10.1016/s1388-2457(98)00050-9

Komi PV, Tesch P (1979) EMG frequency spectrum, muscle structure, and fatigue during dynamic contractions in man. Eur $\mathbf{J}$ Appl Physiol Occup Physiol 42(1):41-50

Lamperti C, Fabbri G, Vercelli L, D’Amico R, Frusciante R, Bonifazi E, Fiorillo C, Borsato C, Cao M, Servida M, Greco F, Di Leo R, Volpi L, Manzoli C, Cudia P, Pastorello E, Ricciardi L, Siciliano G, Galluzzi G, Rodolico C, Santoro L, Tomelleri G, Angelini C, Ricci E, Palmucci L, Moggio M, Tupler R (2010) A standardized clinical evaluation of patients affected by facioscapulohumeral muscular dystrophy: The FSHD clinical score. Muscle Nerve 42(2):213-217. https://doi.org/10.1002/ mus. 21671

Lassche S, Voermans NC, van der Pijl R, van den Berg M, Heerschap A, van Hees H, Kusters B, van der Maarel SM, Ottenheijm CAC, van Engelen BGM (2020) Preserved single muscle fiber specific force in facioscapulohumeral muscular dystrophy. Neurology 94(11):e1157-e1170. https://doi.org/10.1212/WNL.00000 00000008977

Lemmers RJ, Tawil R, Petek LM, Balog J, Block GJ, Santen GW, Amell AM, van der Vliet PJ, Almomani R, Straasheijm KR, Krom YD, Klooster R, Sun Y, den Dunnen JT, Helmer Q, Donlin-Smith CM, Padberg GW, van Engelen BG, de Greef JC, Aartsma-Rus AM, Frants RR, de Visser M, Desnuelle C, Sacconi S, Filippova GN, Bakker B, Bamshad MJ, Tapscott SJ, Miller DG, van der Maarel SM (2012) Digenic inheritance of an SMCHD1 mutation and an FSHD-permissive D4Z4 allele causes facioscapulohumeral muscular dystrophy type 2. Nat Genet 44(12):1370-1374. https:// doi.org/10.1038/ng.2454

Lemmers RJ, van der Vliet PJ, van der Gaag KJ, Zuniga S, Frants RR, de Knijff P, van der Maarel SM (2010) Worldwide population analysis of the $4 \mathrm{q}$ and $10 \mathrm{q}$ subtelomeres identifies only four discrete interchromosomal sequence transfers in human evolution. Am J Hum Genet 86(3):364-377. https://doi.org/10.1016/j. ajhg.2010.01.035

Lindström L, Kadefors R, Petersén I (1977) An electromyographic index for localized muscle fatigue. J Appl Physiol 43(4):750-754. https://doi.org/10.1152/jappl.1977.43.4.750

Linssen WH, Stegeman DF, Joosten EM, van't Hof MA, Binkhorst RA, Notermans SL (1993) Variability and interrelationships of surface EMG parameters during local muscle fatigue. Muscle Nerve 16(8):849-856. https://doi.org/10.1002/mus.880160808

Masuda T, Miyano H, Sadoyama T (1983) The propagation of motor unit action potential and the location of neuromuscular junction investigated by surface electrode arrays. Electroencephalogr Clin Neurophysiol 55(5):594-600. https://doi.org/10.1016/00134694(83)90171-2
Meldrum D, Cahalane E, Conroy R, Fitzgerald D, Hardiman O (2007) Maximum voluntary isometric contraction: reference values and clinical application. Amyotroph Lateral Scler 8(1):47-55

Merletti R, Farina D (2016) Surface electromyography: physiology, engineering, and applications. John Wiley \& Sons

Merletti R, Knaflitz M, De Luca CJ (1990) Myoelectric manifestations of fatigue in voluntary and electrically elicited contractions. J Appl Physiol 69(5):1810-1820. https://doi.org/10.1152/jappl .1990 .69 .5 .1810

Merletti R, Lo Conte LR, Orizio C (1991) Indices of muscle fatigue. J Electromyogr Kinesiol 1(1):20-33. https://doi.org/10.1016/10506411(91)90023-X

Merletti R, Lo Conte LR (1997) Surface EMG signal processing during isometric contractions. J Electromyogr Kinesiol 7(4):241-250. https://doi.org/10.1016/s1050-6411(97)00010-2

Mesin L, Cescon C, Gazzoni M, Merletti R, Rainoldi A (2009) A bidimensional index for the selective assessment of myoelectric manifestations of peripheral and central muscle fatigue. J Electromyogr Kinesiol 19(5):851-863. https://doi.org/10.1016/j.jelek in.2008.08.003

Mesin L, Dardanello D, Rainoldi A, Boccia G (2016) Motor unit firing rates and synchronisation affect the fractal dimension of simulated surface electromyogram during isometric/isotonic contraction of vastus lateralis muscle. Med Eng Phys 38(12):1530-1533. https ://doi.org/10.1016/j.medengphy.2016.09.022

Mul K, Lassche S, Voermans NC, Padberg GW, Horlings CG, van Engelen BG (2016) What's in a name? The clinical features of facioscapulohumeral muscular dystrophy. Pract Neurol 16(3):201207. https://doi.org/10.1136/practneurol-2015-001353

Mul K, Vincenten SCC, Voermans NC, Lemmers R, van der Vliet PJ, van der Maarel SM, Padberg GW, Horlings CGC, van Engelen BGM (2017) Adding quantitative muscle MRI to the FSHD clinical trial toolbox. Neurology 89(20):2057-2065. https://doi. org/10.1212/WNL.0000000000004647

Naumann M, Reiners K (1996) Diagnostic value of in situ muscle fiber conduction velocity measurements in myopathies. Acta Neurol Scand 93(2-3):193-197. https://doi. org/10.1111/j.1600-0404.1996.tb00198.x

Olsen DB, Gideon P, Jeppesen TD, Vissing J (2006) Leg muscle involvement in facioscapulohumeral muscular dystrophy assessed by MRI. J Neurol 253(11):1437-1441. https://doi.org/10.1007/ s00415-006-0230-z

Place N, Millet GY (2020) Quantification of neuromuscular fatigue: what do we do wrong and why? Sports Med 50(3):439-447. https ://doi.org/10.1007/s40279-019-01203-9

Rainoldi A, Bullock-Saxton JE, Cavarretta F, Hogan N (2001) Repeatability of maximal voluntary force and of surface EMG variables during voluntary isometric contraction of quadriceps muscles in healthy subjects. J Electromyogr Kinesiol 11(6):425-438

Rampichini S, Vieira TM, Castiglioni P, Merati G (2020) Complexity analysis of surface electromyography for assessing the myoelectric manifestation of muscle fatigue: a review. Entropy 22(5):529. https://doi.org/10.3390/e22050529

Ricci G, Ruggiero L, Vercelli L, Sera F, Nikolic A, Govi M, Mele F, Daolio J, Angelini C, Antonini G, Berardinelli A, Bucci E, Cao M, D’Amico MC, D’Angelo G, Di Muzio A, Filosto M, Maggi L, Moggio M, Mongini T, Morandi L, Pegoraro E, Rodolico C, Santoro L, Siciliano G, Tomelleri G, Villa L, Tupler R (2016) A novel clinical tool to classify facioscapulohumeral muscular dystrophy phenotypes. J Neurol 263(6):1204-1214. https://doi. org/10.1007/s00415-016-8123-2

Rijken NH, van der Kooi EL, Hendriks JC, van Asseldonk RJ, Padberg GW, Geurts AC, van Engelen BG (2014) Skeletal muscle imaging in facioscapulohumeral muscular dystrophy, pattern and asymmetry of individual muscle involvement. Neuromuscul Disord 24(12):1087-1096. https://doi.org/10.1016/j.nmd.2014.05.012 
Sanders DB, Stålberg EV, Nandedkar SD (1996) Analysis of the electromyographic interference pattern. J Clin Neurophysiol 13(5):385-400

Schillings ML, Kalkman JS, Janssen HM, van Engelen BG, Bleijenberg G, Zwarts MJ (2007) Experienced and physiological fatigue in neuromuscular disorders. Clin Neurophysiol 118(2):292-300. https://doi.org/10.1016/j.clinph.2006.10.018

Schipper K, Bakker M, Abma T (2017) Fatigue in facioscapulohumeral muscular dystrophy: a qualitative study of people's experiences. Disabil Rehabil 39(18):1840-1846. https://doi.org/10.1080/09638 288.2016.1212109

Schulte-Mattler WJ, Muller T, Deschauer M, Gellerich FN, Iaizzo PA, Zierz S (2003) Increased metabolic muscle fatigue is caused by some but not all mitochondrial mutations. Arch Neurol 60(1):5058. https://doi.org/10.1001/archneur.60.1.50

Siciliano G, Schirinzi E, Simoncini C, Ricci G (2019) Exercise therapy in muscle diseases: open issues and future perspectives. Acta Myol 38(4):233-238

Tasca G, Monforte M, Iannaccone E, Laschena F, Ottaviani P, Leoncini E, Boccia S, Galluzzi G, Pelliccioni M, Masciullo M, Frusciante R, Mercuri E, Ricci E (2014) Upper girdle imaging in facioscapulohumeral muscular dystrophy. PLoS ONE 9(6):e100292. https:// doi.org/10.1371/journal.pone.0100292

Tawil R (2018) Facioscapulohumeral muscular dystrophy. Handb Clin Neurol 148:541-548. https://doi.org/10.1016/B978-0-444-64076 $-5.00035-1$

Tupler R, Gabellini D (2004) Molecular basis of facioscapulohumeral muscular dystrophy. Cell Mol Life Sci 61(5):557-566. https://doi. org/10.1007/s00018-003-3285-3

Turki A, Hayot M, Carnac G, Pillard F, Passerieux E, Bommart S, Raynaud de Mauverger E, Hugon G, Pincemail J, Pietri S, Lambert K, Belayew A, Vassetzky Y, Juntas Morales R, Mercier J, Laoudj-Chenivesse D (2012) Functional muscle impairment in facioscapulohumeral muscular dystrophy is correlated with oxidative stress and mitochondrial dysfunction. Free Radic Biol Med 53(5):1068-1079. https://doi.org/10.1016/j.freeradbio med.2012.06.041

van Deutekom JC, Wijmenga C, van Tienhoven EA, Gruter AM, Hewitt JE, Padberg GW, van Ommen GJ, Hofker MH, Frants RR (1993) FSHD associated DNA rearrangements are due to deletions of integral copies of a $3.2 \mathrm{~kb}$ tandemly repeated unit. Hum Mol Genet 2(12):2037-2042. https://doi.org/10.1093/hmg/2.12.2037

Vandebrouck C, Imbert N, Constantin B, Duport G, Raymond G, Cognard C (2002) Normal calcium homeostasis in dystrophinexpressing facioscapulohumeral muscular dystrophy myotubes. Neuromuscul Disord 12(3):266-272

Vercoulen JH, Swanink CM, Fennis JF, Galama JM, van der Meer JW, Bleijenberg G (1994) Dimensional assessment of chronic fatigue syndrome. J Psychosom Res 38(5):383-392. https://doi. org/10.1016/0022-3999(94)90099-x

Viitasalo JH, Komi PV (1977) Signal characteristics of EMG during fatigue. Eur J Appl Physiol Occup Physiol 37(2):111-121. https ://doi.org/10.1007/BF00421697

Wijmenga C, Hewitt JE, Sandkuijl LA, Clark LN, Wright TJ, Dauwerse HG, Gruter AM, Hofker MH, Moerer P, Williamson R et al (1992) Chromosome 4q DNA rearrangements associated with facioscapulohumeral muscular dystrophy. Nat Genet 2(1):26-30. https://doi.org/10.1038/ng0992-26

Zwarts MJ, Arendt-Nielsen L (1988) The influence of force and circulation on average muscle fibre conduction velocity during local muscle fatigue. Eur J Appl Physiol Occup Physiol 58(3):278-283. https://doi.org/10.1007/BF00417263

Publisher's Note Springer Nature remains neutral with regard to jurisdictional claims in published maps and institutional affiliations. 